\title{
Idiopathic portal hypertension associated with cytotoxic drugs
}

\author{
P Shepherd, D J Harrison
}

\begin{abstract}
Four patients developed clinically important portal hypertension with histological features of idiopathic portal hypertension while they were receiving cytotoxic drugs for chronic myeloid leukaemia and Hodgkin's disease. Mild sclerosis of some small portal triads was the only abnormality seen at light microscopical examination in three of the four cases. In the remaining case light microscopical findings seemed to be normal. Two cases examined by electron microscopy showed perisinusoidal fibrosis; in one case this was the only abnormality detected.

There is an association between idiopathic portal hypertension and the use of chemotherapeutic agents, particularly thioguanine. Adequate histological examination of liver tissue, including electron microscopic studies, is recommended for patients who develop hepatic problems while receiving cytotoxic treatment to elucidate this problem.
\end{abstract}

Idiopathic portal hypertension in the absence of cirrhosis or other demonstrable causes of portal hypertension is uncommon, accounting for $3-4 \%$ of all cases in Western countries ${ }^{1}$ but $25-30 \%$ in India ${ }^{2}$ and Japan. ${ }^{3}$ Fibrosis of small portal triads may be present and in most the aetiology is unknown: some have been associated with exposure to arsenic, ${ }^{45}$ vinyl chloride, ${ }^{56}$ and vitamin A. ${ }^{7}$ Only rarely have chemotherapeutic agents been implicated. ${ }^{8}$

Key et al recently attributed non-cirrhotic portal hypertension to nodular regenerative hyperplasia (NRH) in four patients with chronic myeloid leukaemia who were treated with busulphan and thioguanine. ${ }^{9} \mathrm{NRH}$ is rare and may be difficult to diagnose on small needle biopsy specimens. ${ }^{10}$ In reported series of patients with $\mathrm{NRH}^{112}$ mild fibrosis of small portal tracts was noted in most, and it has been suggested that the regenerative nodules may develop as a response to obliterative portal venopathy. ${ }^{11}$

We report the development of portal hypertension in four patients after treatment of chronic myeloid leukaemia and Hodgkin's disease with chemotherapeutic drugs. Mild portal sclerosis was present in three of four cases, and in one other the only apparent abnormality was perisinusoidal fibrosis seen on electron microscopical examination.

\section{Case reports}

CASE 1

A 55 year old woman developed chronic myeloid leukaemia (CML) in July 1982. Chromosomal analysis showed the typical t9;22 translocation. The spleen was palpable $7 \mathrm{~cm}$ below the costal margin, but the liver was not enlarged and liver function tests at diagnosis were normal. She was entered into the Medical Research Council trial of treatment for chronic myeloid leukamia (MRC CML II). Over the next 17 months she received busulphan (total dose $442 \mathrm{mg}$ ) and thioguanine (total dose $17.5 \mathrm{~g}$ ) with symptomatic control of her disease and resolution of splenomegaly. The results of the liver function tests are shown in fig 1 . In December 1983 busulphan and thioguanine were discontinued because of possible pulmonary toxicity. In February 198421 months after the start of chemotherapy she developed gastrointestinal haemorrhage secondary to oesophageal varices. These were treated by sclerotherapy. Control of her CML was subsequently maintained with hydroxyurea. In May 1984 she had further variceal haemorrhage which was treated by oesophageal transection. A wedge liver biopsy specimen was his-
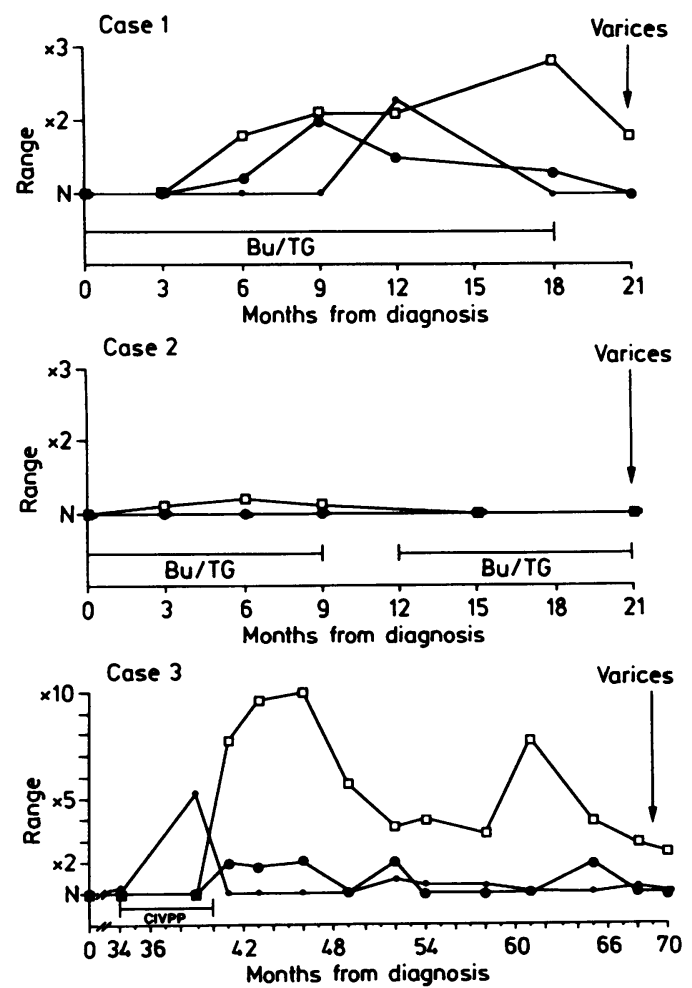

Figure 1 Liver function tests showing degree of abnormalities present before onset of variceal haemorrhage. $N=$ normal; $\square=$ alkaline phosphatase; $\boldsymbol{O}=$ alanine transferase; $\bullet=$ bilirubin. 
tologically normal. Angiography showed no evidence of hepatic or portal vein thrombosis. Over the next year she suffered recurrent variceal bleeding and developed ascites. Three months later blast transformation occurred and she died shortly after.

CASE 2

A 55 year old man developed chronic myeloid leukaemia in March 1983. The Philadelphia chromosome, however, was not present. The spleen was palpable $4 \mathrm{~cm}$ below the costal margin, but the liver was not enlarged and liver function tests were normal. He was entered into the MRC CML II trial and received busulphan (total dose $252 \mathrm{mg}$ ) and thioguanine $(10 \cdot 1 \mathrm{~g})$ with good control of his disease. Serial liver function tests are shown in figure 1. In December 1983 he developed haemoatemesis and malaena due to a bleeding gastric ulcer, seen on endoscopic examination. Chemotherapy was stopped and cimetidine started. Repeat endoscopy in March 1984 was normal. In September 1984, due to increasing leucocyte counts, busulphan and thioguanine were restarted. In December 198421 months after starting treatment haematemesis recurred and another endoscopy showed bleeding oesophageal varices; these were treated with sclerotherapy. Chemotherapy was continued until September 1985 when recurrent variceal haemorrhage uncontrolled by sclerotherapy required laparotomy and removal of the spleen. Portal venography showed increased pressure in the portal vein but no other abnormality. In particular, there was no evidence of obstruction of the portal tract. Chemotherapy was changed to hydroxyurea and since then he has had no further problems.
CASE 3

A 34 year old woman presented in 1981 with cervical lymphadenopathy. Biopsy and staging showed nodular sclerosing Hodgkin's disease (stage IIA). She was treated with radiotherapy with regression of the disease. In March 1985 she developed recurrent Hodgkin's disease in the lungs. Liver function tests and a barium swallow at this time were normal. She was treated with combination chemotherapy using chlorambucil, vinblastine, procarbazine and prednisolone (CIVPP) for six courses with regression of her pulmonary disease. Shortly afterwards she developed pronounced abnormalities of liver function tests, particularly alkaline phosphatase (figure 1). Her liver was not enlarged and an open liver biopsy specimen was obtained. Because of the severely increased alkaline phosphatase activity an endoscopic retrograde cholangiopancreatogram was performed to assess the biliary tree and pancreas. Mild deformity of the intrahepatic ducts in the left lobe of the liver was seen but no other abnormality. Spider naevi were noted and a barium swallow in February 1988 showed oesophageal varices.

\section{CASE 4}

Chronic myeloid leukaemia was diagnosed in a 68 year old woman in December 1986..She was entered into the MRC CML II trial and received busulphan (total dose $550 \mathrm{mg}$ ) and thioguanine (total dose $23.6 \mathrm{~g}$ ) until May 1988 when thioguanine was stopped because of concern about potential hepatotoxicity associated with its use. At diagnosis slightly increased transaminase activities were present $(<2 \times \mathrm{N})$ which persisted along with a mild increase in alkaline phosphatase during treatment. Busul-
Figure 2 Portal venography necropsy: Dye was injected into the portal vein filling the liver with retrograde filling of the spleen. The spleen shows normal capillary filling but portal vein radicals are nipped off at the venular level.

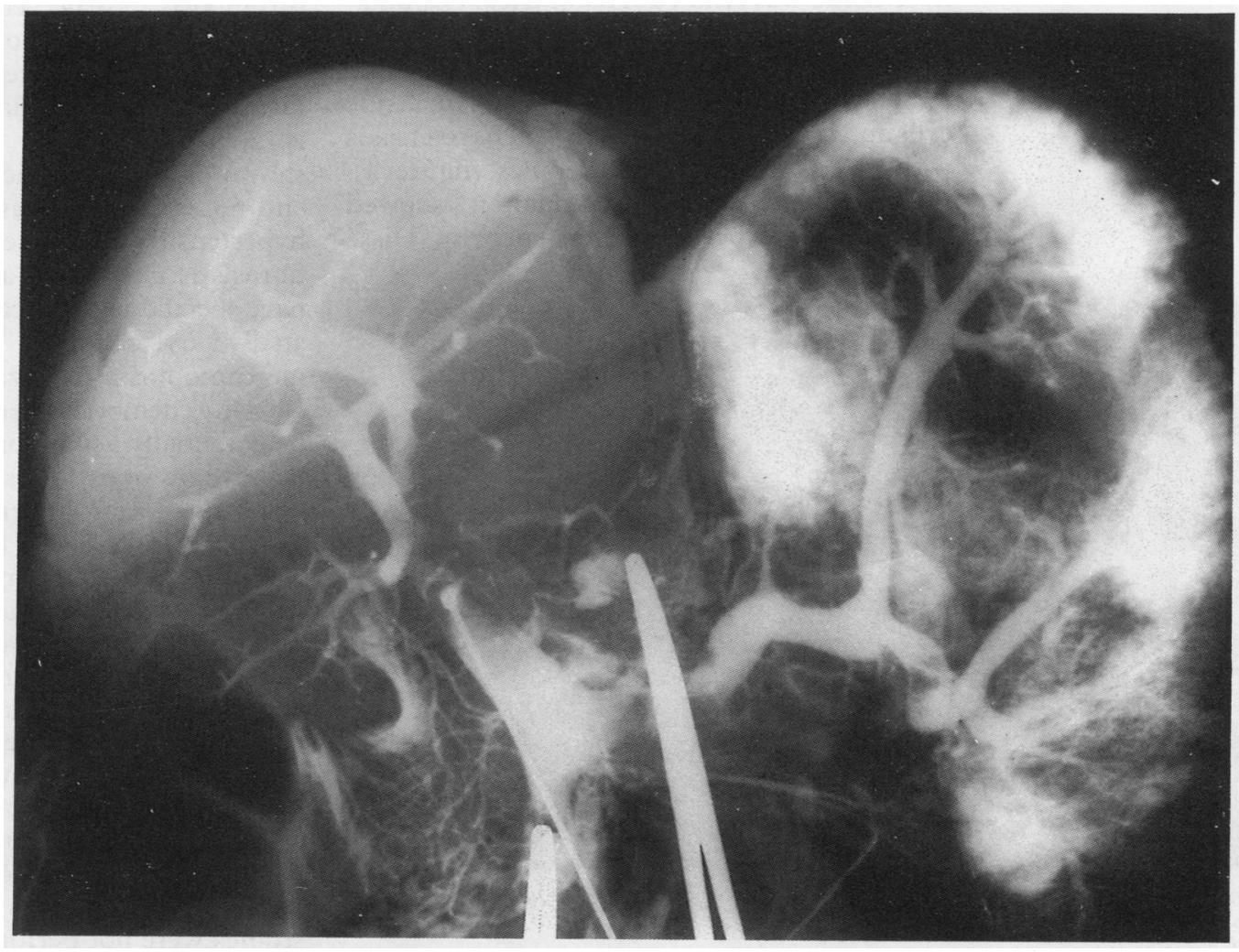




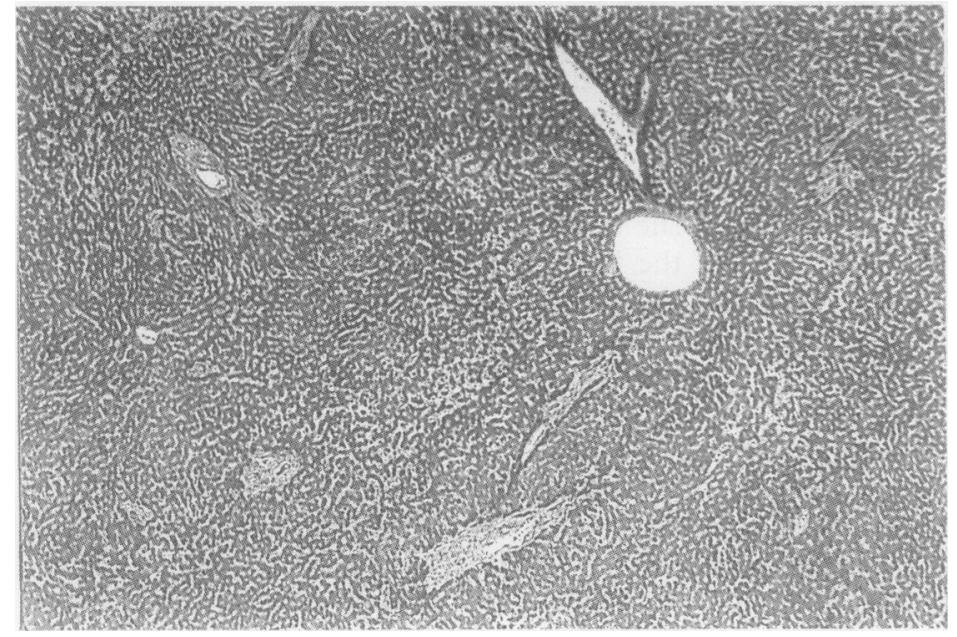

Figure 3 Photomicrograph showing normal lobular architecture (Masson Trichrome).

phan was continued and she remained well until September 1988 when a bleeding duodenal ulcer, which was confirmed by endoscopy, developed. In January 1989 she developed jaundice, ascites, and hepatosplenomegaly. A bone marrow aspirate still showed chronic phase features but chromosomal examination showed an isochromosome 17, suggesting transformation to a more acute phase. Haematemesis developed and endoscopic examination showed bleeding oesophageal varices and duodenal ulceration. She died in February 1989.

\section{Pathology}

CASE 1

At necropsy a portal venogram showed delayed filling with "cut off" of small portal vein radicles but not evidence of thrombosis (figure 2). This appearance was not seen when control liver tissue was studied. Histological examination of the liver before death and at necropsy had shown normal lobular architecture (figure 3). The sinusoids were infiltrated by leukaemic cells and there was fibrosis of small portal triads with narrowing of portal venules (figure 4). Electron microscopic examination showed fibrosis in the perisinusoidal space of Disse (figure 5).

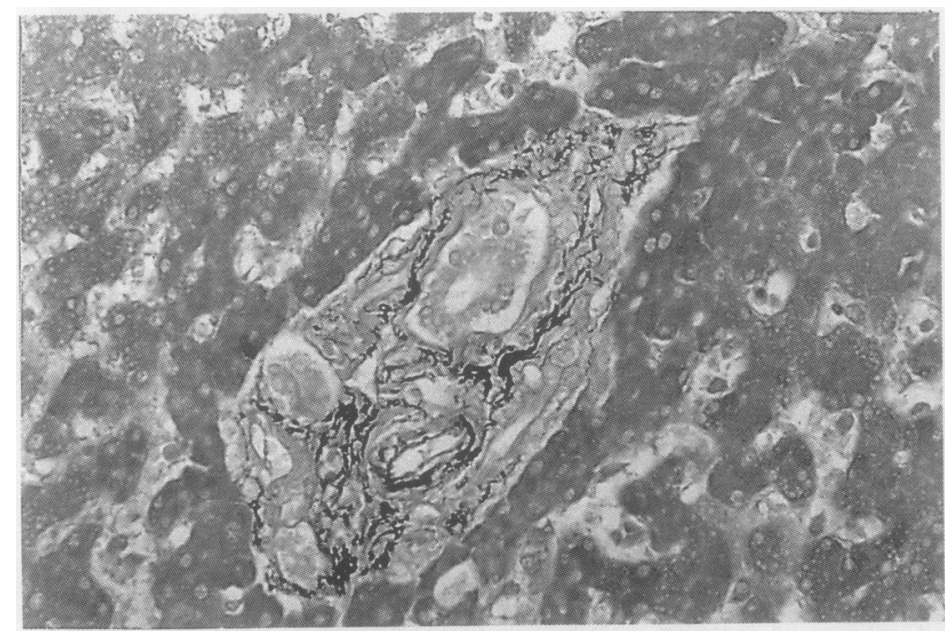

Figure 4 Photomicrograph of portal triad containing bile ducts and nepatzc artery but no distinguishable portal vein. There is an increase in portal fibrosis (Orcein Shikata).
CASE 2

The liver biopsy specimen at laparotomy showed normal architecture with no evidence of leukaemic infiltration of sinusoids. There was mild sclerosis of small portal triads similar to that seen in figure 4 .

CASE 3

A liver biopsy specimen seemed to be normal on light microscopical examination. Electron microscopic examination showed perisinusoidal fibrosis in the space of Disse, similar to that seen in figure 5 .

\section{CASE 4}

At necropsy prominent oesophageal varices were present. No abnormalities were seen in the portal vein, hepatic vein, or hepatic artery. Histological sections showed that liver architecture was well preserved without cirrhosis, parenchymal fibrosis, or nodular regenerative hyperplasia. Many portal triads were normal but some showed increased fibrosis with narrowing and occlusion of portal vein branches. The appearances were those of hepatoportal sclerosis.

\section{Discussion}

We have described four patients who developed non-cirrhotic portal hypertension associated with the use of cytotoxic agents. Studies of series of patients with idiopathic portal hypertension and comparison with similar entities designated as hepatoportal sclerosis and non-cirrhotic portal fibrosis have shown that they describe similar conditions, characterised by variable degrees of portal tract fibrosis without cirrhosis on histological examination. ${ }^{1-3513-15}$ Angiographic abnormalities with "cut off" of small portal veins were seen without evidence of portal vein thrombosis. ${ }^{5}$ Portal venography carried out at necropsy on case 1 showed delayed filling and "cut-off" of portal vein radicles, apparently identical with the findings of Villeneuve. ${ }^{5}$ This is attributed to the perisinusoidal fibrosis shown on electron microscopical examination and noted in other cases of idiopathic portal hypertension. ${ }^{2816}$ "The pathogenesis of the fibrosis is unknown-and in only a few cases have specific aetiological agents been incriminated-vinyl chloride, ${ }^{56}$ arsenic, ${ }^{45}$ vitamin $A,{ }^{7}$ mercaptopurine and azathioprine. ${ }^{8}$

Many chemotherapeutic agents are known to be potentially hepatotoxic and may cause acute or chronic hepatic lesions. ${ }^{1718}$ The development of portal hypertension without light microscopic evidence of liver injury such as cirrhosis, necrosis, chronic active hepatitis or vascular obstruction (idiopathic portal hypertension), however, is very unusual. Nataf described two patients treated with continuous azathioprine and mercaptopurine after renal transplantation who developed idiopathic portal hypertension without histological lesions at light microscopy but with perisinusoidal fibrosis at electron microscopy. ${ }^{8}$ These cases seem to be similar to two of ours. In the remaining two cases electron microscopic studies were not performed. In one of Nataf's 
Figure 5 Electron micrograph showing bundles of striated collagen between endothelium and hepatocyte in the space of Disse.

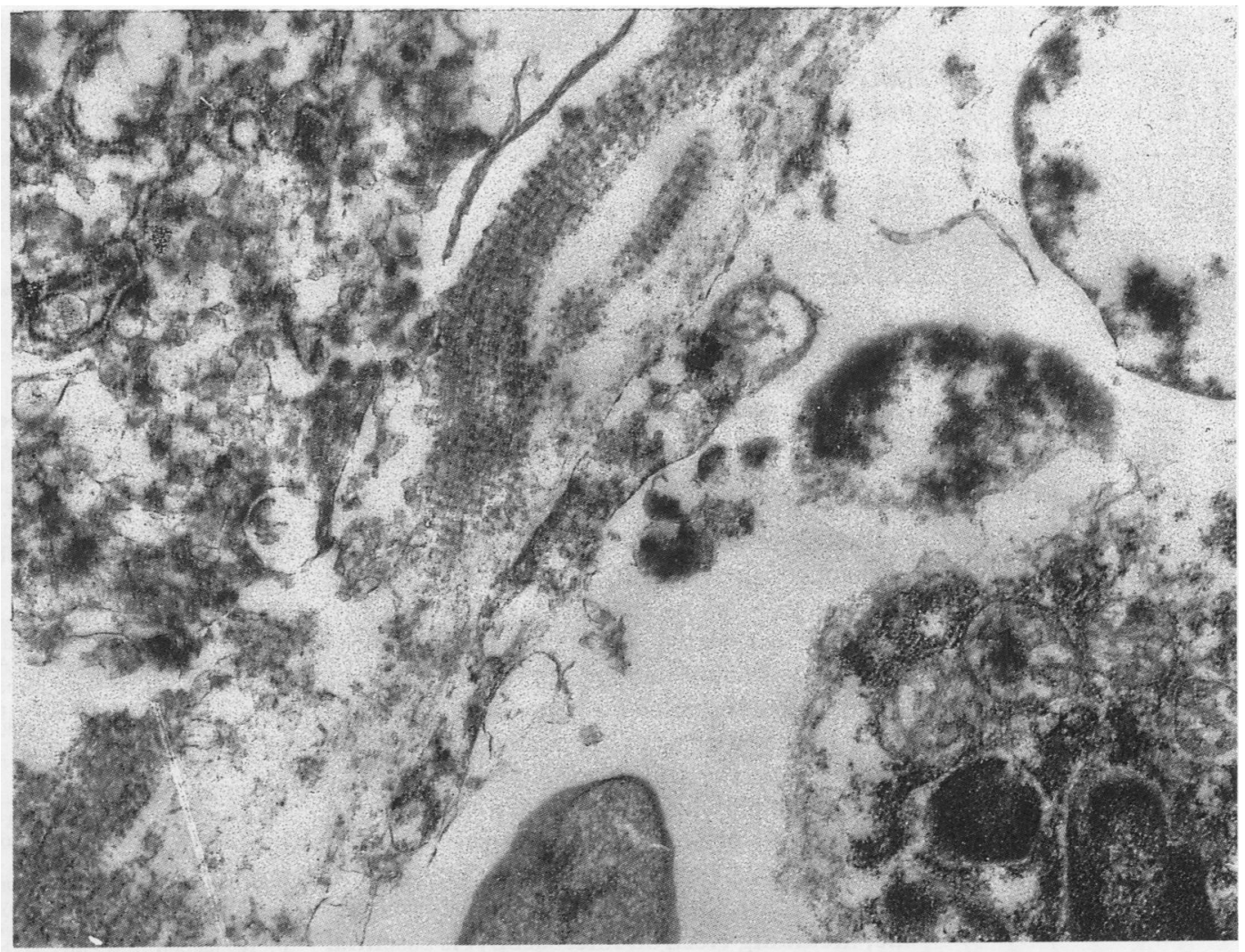

cases serial biopsy specimens showed progression to hepatoportal sclerosis, suggesting that this may be a subsequent manifestation of fibroblastic proliferation.

Key et al have recently described five patients with chronic myeloid leukaemia, treated with busulphan and thioguanine, who developed portal hypertension with oesophageal varices. ${ }^{9}$ Histological findings for four showed small intrahepatic nodules, interpreted as nodular regenerative hyperplasia, a rare cause of portal hypertension. This is a difficult diagnosis to make because of the limited material available: indeed it may be impossible to establish without a wedge biopsy. ${ }^{10}$ Evaluation of series of patients with nodular regenerative hyperplasia has shown mild fibrosis affecting the portal tracts in most, ${ }^{11}{ }^{12}$ and it has been postulated that the hyperplastic nodules arise as a secondary response to obliterative portal venopathy. ${ }^{11}$ There may therefore be an association between this disorder and other conditions associated with fibrosis of portal tracts.

In the Medical Research Council's second therapeutic trial in chronic myeloid leukaemia (MRC CML II trial) in which single agent treatment (busulphan) was compared with combination treatment (busulphan and thioguanine) 18 cases of oesophageal varices occurred during the chronic phase of the disease (personal observation, CML Trial Office, Edinburgh). All 18 cases of varices occurred in patients receiving combination treatment, strongly suggesting that thioguanine was asssociated with the liver injury. These include four of the five patients reported by $\mathrm{Key}^{9}$ and cases 1,2 , and 4 described here. Thioguanine has been implicated in hepatitis and venoocclusive disease of the liver, ${ }^{17}$ but in none of these seven patients for whom biopsy specimens were available were these findings seen. Thus seven out of these $18 \mathrm{MRC}$ CML trial patients had non-cirrhotic portal hypertension. The association between the use of combination treatment and the development of portal hypertension has led to its withdrawal from the MRC CML II trial.

This association with chronic myeloid leukaemia and the use of thioguanine is particularly interesting. A similar picture has not been described after the use of thioguanine or a related drug, mercaptopurine, in the treatment of other leukaemias, such as acute myeloid leukaemia or acute lymphoblastic leukaemia where the drug can be used in a similar manner during maintenance treatment. Portal hypertension may be seen at diagnosis in chronic myeloid leukaemia related to leukaemic infiltration of sinusoids. ${ }^{19}$ Perhaps residual infiltration, despite apparent good control of disease, may render these patients more vulnerable to this condition, or perhaps the combination with busulphan is uniquely dangerous? Another factor that may predispose to toxicity is the continuous use of chemotherapeutic agents, as is the case in CML. As shown in case 3 , however, this has also occurred with intermittent courses of treatment.

Sclerosis of portal tracts has also been found at necropsy without evidence of liver disease. Its cause and potential clinical importance are unclear. ${ }^{20}$ What is clear from reported cases, however, is that clinically important portal hypertension can result from this kind of lesion. We believe, along with others, that fibrosis is a reaction to injury by a variety of identified and unidentified toxins and is responsible for the syndrome of "idiopathic portal hypertension". ${ }^{25}$ Fibrosis may be minimal or 
absent when assessed at light microscopy, but perisinusoidal fibrosis visible only by electron microscopy may be associated with clinically important portal hypertension.

We draw attention to its association with chemotherapeutic agents, particularly thioguanine, but also, as shown in case 3, other cytotoxic agents. Of the drugs given in case 3 , chlorambucil may be the most likely offender.

The paucity of previously reported cases of idiopathic portal hypertension in association with chemotherapeutic agents suggests that this is the result of a rare idiosyncratic reaction to the drugs. Its incidence, however, may be underestimated if liver biopsy or necropsy material is not obtained or adequately evaluated in any such patient who develops evidence of liver dysfunction. It is not known whether the lesions can regress with time. In one case associated with exposure to vinyl chloride a second biopsy specimen obtained two years later showed persistent fibrosis. ${ }^{6}$ Liver function tests are not particularly helpful in screening for this disorder; they may be normal or only slightly abnormal as in two of our cases. Portal venography showing the characteristic "cut off" may be useful. Adequate histological examination of liver tissue, including electron microscopy, is essential for evaluating possible injury in cases of portal hypertension or unexplained hepatic dysfunction associated with the use of these agents, and will be required to determine more clearly the incidence and pathogenesis of this disorder.

We are grateful to Drs N C Allan, H M Cameron, N C Finlayson, E J Gaminara, R D Hyde and A C Parker for permission to report these cases, and to Anne Young for typing.
1 Iber FL. Portal hypertension in the presence of normal liver morphology. Ann N Y Acad Sci 1970;170:115-26.

2 Sama SK, Bhargava S, Gopi Nath N, et al. Non-cirrhotic portal fibrosis. Am J Med 1971;51:160-9.

3 Okuda K, Nakashima T, Okudaira M, et al. Liver pathology of idiopathic portal hypertension. Comparison with nonof idiopathic portal hypertension. Comparison with non-
cirrhotic portal fibrosis of India. The Japan idiopathic portal hypertension study. Liver 1982;2:176-92.

4 Morris JS, Schmid M, Newman S, et al. Arsenic and noncirrhotic portal hypertension. Gastroenterology 1974;64:86-94.

5 Villeneuve JP, Huet PM, Joly JG, et al. Idiopathic portal hypertension. Am J Med 1976;61:459-64.

6 Thomas LB, Popper H, Berk PD, et al. Vinyl chloride induced liver disease. From idiopathic portal hypertension (Banti's syndrome) to angiosarcomas. $N$ Engl J Med 1975;292:17-22.

7 Russell RM, Boyer JL, Bagheri SA, Hruban Z. Hepatic injury from chronic hypervitaminosis A resulting in portal hypertension and ascites. N Engl J Med 1974;291:435-40.

8 Nataf C, Feldmann G, Lebrec D, et al. Idiopathic portal hypertension (perisinusoidal fibrosis) after renal transplantation. Gut 1979;20:531-7.

9 Key NS, Emerson PM, Allan NC, et al. Oesophageal varices associated with busulphan-thioguanine combination therapy for chronic myeloid leukaemia. Lancet 1987;ii:1050-2.

10 Scheuer PJ. Liver biopsy interpretation. Third Edition. London: Baillière Tindall, 1980.

11 Wanless IR, Godwin TA, Allen F, Feder A. Nodular regenerative hyperplasia of the liver in haematologic disorders: a possible response to obliterative portal venopathy. Medicine 1980;59:367-79.

12 Stromeyer FW, Ishak KG. Nodular transformation (nodular 'regenerative' hyperplasia) of the liver. Hum Pathol 1981;12:60-71.

13 Aikat BK, Brunsnurmath SR, Chautiani PN, et al. The pathology of non cirrhotic portal fibrosis. Hum Pathol 1979;10:405-18.

14 Mikkelsen WP, Edmondson HA, Peters RL, et al. Extra and intrahepatic portal hypertension without cirrhosis intrahepatic portal hypertension without cirrho
(hepatoportal sclerosis). Ann Surg 1965;162:602-20.

15 Benhamou JP, Lebrec D. Non-cirrhotic intrahepatic porta hypertension in adults. Clin Gastroenterol 1985;14:21-31.

16 Tandon BN, Lakshminarayanan R, Bhargava S, et al. Ultrastructure of the liver in non-cirrhotic portal fibrosis with portal hypertension. Gut 1970;11:905.

7 Zimmerman HJ. Hepatotoxic effects of oncotherapeutic agents. Progr Liver Dis 1986;8:621-42.

18 Menard DB, Gisselbrecht MM, Marty M, et al. Antineoplastic agents and the liver. Gastroenterology 1980;78: 142-64.

19 Datta DV, Grover SL, Saini VK, et al. Portal hypertension in chronic leukaemia. Br J Haematol 1975;31:279-85.

20 Wanless IR, Bernier V, Seger M. Intrahepatic portal vein sclerosis in patients without a history of liver disease. An autopsy study. Am J Pathol 1982;106:63-70. 